\title{
Crescimento inicial e diagnose nutricional de plantas de milho cultivadas com omissão de macronutrientes em Argissolo
}

\author{
Janderson Carmo LIMA ${ }^{1}$, Marilza Neves NASCIMENTO ${ }^{1}$, Railda Santos JESUS², \\ Alismário Leite SILVA ${ }^{3}$, Anacleto Ranulfo SANTOS ${ }^{4}$, Uasley Caldas OLIVEIRA ${ }^{3}$
}

${ }^{1}$ Universidade Estadual de Feira de Santana, Feira de Santana, BA, Brasil. (Orcid: *; 0000-0003-3344-9106)

2Programa de Pós-Graduação em Ciências Agrárias, Universidade Federal do Recôncavo Baiano, Cruz das Almas, BA, Brasil. (Orcid: 0000-0003-1029-9774)

3 Programa de Pós-Graduação em Recursos Genéticos Vegetais, Universidade Estadual de Feira de Santana, Feira de Santana, BA, Brasil. (Orcid: 0000-0001-7541-5292; 0000-0001-6551-7746)

${ }^{4}$ Centro de Ciências Agrárias, Ambientais e Biológicas, Universidade Federal do Recôncavo Baiano, Cruz das Almas, BA, Brasil. (Orcid: 0000-0003-4629-3948)

*E-mail: janderson_ufrb@yahoo.com.br (Orcid: 0000-0002-7564-5044)

Recebido em 10/09/2019; Aceito em 16/08/2020; Publicado em 11/11/2020.

\begin{abstract}
RESUMO: O objetivo do trabalho foi avaliar o crescimento inicial e diagnose nutricional de plantas de milho cultivadas com omissão de macronutrientes em Argissolo. O experimento foi desenvolvido em estufa, tendo como substrato Argissolo amarelo distrocoeso, utilizou-se vasos com capacidade de $3 \mathrm{dm}^{3}$, tendo como tratamentos: Solo sem adubação, Adubação referência e omissão de nitrogênio $(\mathrm{N})$, fósforo $(\mathrm{P})$ e potássio $(\mathrm{K})$ respectivamente, mantendo cinco repetições por tratamento, totalizando 25 unidades experimentais, em delineamento inteiramente casualizado. Foi realizado o plantio via propagação sexuada e após uma semana foi feito o desbaste, deixando apenas uma planta por vaso. Aos 30 dias após a aplicação dos tratamentos, foram avaliados os parâmetros de crescimento: altura das plantas, número de folhas, diâmetro do colmo, massa seca (MS) da parte aérea (PA) e raiz (R), além das determinações nutricionais: concentração de nitrogênio, fósforo e potássio na PA e R. Os resultados encontrados foram submetidos a análise de variância e posteriormente ao teste de medias de acordo a significância. O crescimento inicial das plantas de milho foi severamente comprometido com a ausência dos nutrientes $\mathrm{N}$ e P, destacando assim a importância dessa suplementação. A diagnose nutricional mostrou que a omissão dos macronutrientes comprometeu o acúmulo desses elementos. Palavras-chave: gramíneas; fertilidade do solo; nutrição mineral de plantas; Zea mays.
\end{abstract}

\section{Initial growth and nutritional diagnosis of maize plants cultivated with omission of macronutrients in argissolo}

\begin{abstract}
The objective of this work was to evaluate the initial growth and nutritional diagnosis of corn plants cultivated with omission of macronutrients in Argisol. The experiment was carried out in a greenhouse with subsoil yellow dystrocoes, using pots with a capacity of $3 \mathrm{dm}^{3}$, using as treatments: Soil without fertilization, reference fertilization and omission of nitrogen $(\mathrm{N})$, phosphorus $(\mathrm{P})$ and potassium $(\mathrm{K})$, maintaining five replicates per treatment, totaling 25 experimental units in a completely randomized design. Planting was carried out via sexual propagation and after one week the thinning was done, leaving only one plant per pot. At 30 days after application of the treatments, growth parameters were evaluated: plant height, leaf number, shoot diameter, shoot dry mass (AP) and root (R), in addition to the nutritional determinations: nitrogen concentration, phosphorus and potassium in PA and R. The results were submitted to analysis of variance and later to the test of means according to significance. The initial growth of corn plants was severely compromised by the absence of $\mathrm{N}$ and $\mathrm{P}$ nutrients, thus highlighting the importance of this supplementation. The nutritional diagnosis showed that omission of macronutrients compromised the accumulation of these elements.
\end{abstract}

Keywords: grasses; soil fertility; mineral nutrition of plants; Zea mays.

\section{INTRODUÇÃO}

A cultura do milho é considerada uma das principais commodities do agronegócio brasileiro, devido à ampla utilização, seja está para o consumo humano, animal, ou nas indústrias químicas e de biocombustíveis (CONAB, 2018). De acordo com o IBGE (2017), a cultura do milho ocupou cerca de 17 milhões de hectares de área plantada, equivalente a uma produção de 97 milhões de toneladas de grãos, com o rendimento médio de aproximadamente $5.6 \mathrm{t} \mathrm{ha}^{-1}$, destacando a região Centro-Oeste como maior produtora.

A produtividade das culturas depende de vários fatores, desde as condições climáticas, manejo (solo, pragas, doenças, plantas daninhas e irrigação), até as exigências nutricionais que influenciam na capacidade da planta de retirar nutrientes do meio de cultivo. Tais nutrientes apresentam funções essenciais, quando não presentes em quantidades suficientes 
causam limitações nas células promovendo alterações no metabolismo da planta e consequentemente baixa produtividade (MCGRATH et al., 2014).

Os nutrientes minerais são classificados em macro e micronutrientes, de acordo com a concentração encontrada em seus tecidos. $\mathrm{O}$ nitrogênio $(\mathrm{N})$ e o potássio $(\mathrm{K})$ são os nutrientes mais requeridos pela cultura do milho, em seguida o fósforo (P) (GONDIM et al., 2010). O N atua no metabolismo vegetal participando diretamente na biossíntese de proteínas e clorofilas (TAIZ et al., 2017). Em plantas de milho, por exemplo, ocorre uma intensa absorção de $\mathrm{N}$ nas fases iniciais de desenvolvimento, devido a sua intensa atividade metabólica (GONDIM et al., 2016).

$\mathrm{O}$ fósforo $(\mathrm{P})$ é encontrado em formas orgânicas e inorgânicas no solo e este influencia no desenvolvimento do sistema radicular, atua na floração, fecundação, formação e maturação do grão (CARMO et al., 2014). Nas plantas, a carência de fósforo resulta em menor crescimento, redução da expansão de área foliar, no número de folhas e o diâmetro do caule (TAIZ et al., 2017). Já o potássio (K), depois do nitrogênio, é o nutriente mais extraído pelas plantas e está relacionado ao processo fotossintético, é ativador enzimático e atua na translocação dos assimilados para as folhas, abertura e fechamento dos estômatos e regulação osmótica (ELMER; DATNOFF, 2014).

A adubação mineral apresenta papel importante no crescimento e produtividade da maioria das plantas cultivadas. A aplicação de quantidades excessivas dos nutrientes essenciais pode ocasionar sérios danos ao metabolismo vegetal, apresentando uma baixa eficiência no uso dos fertilizantes, além de prejuízos financeiros (ELMER; DATNOFF, 2014). De acordo com Vieira et al. (2014), o método de omissão de nutrientes é uma alternativa rápida para conhecer as exigências nutricionais de uma determinada espécie, visto que a carência nutricional promove uma diminuição na produtividade, sendo esta ocasionada por deficiências ou excessos dos elementos essenciais. Desta forma, o objetivo do trabalho foi avaliar o crescimento inicial e diagnose nutricional de plantas de milho cultivadas com omissão de macronutrientes em Argissolo.

\section{MATERIAL E MÉTODOS}

\subsection{Material vegetal}

As plantas de milho foram produzidas a partir da propagação sexuada (sementes), em viveiro. $\mathrm{O}$ material para propagação (BRS 3046) foi adquirido no comércio local da cidade de Cruz das Almas-Ba.

\subsection{Implantação e delineamento experimental}

O experimento foi desenvolvido no campo experimental do Centro de Ciências Agrárias, Ambientais e Biológicas da Universidade Federal do Recôncavo da Bahia - UFRB, no Município de Cruz das Almas-Bahia (12 $40^{\text {ee }}$; 39 $39^{\circ} 06^{\text {ee }} \mathrm{W} ; 226$ metros de altitude), no período de novembro a dezembro de 2018.

Foram coletadas amostras de um Argissolo distrófico na camada de $0-20 \mathrm{~cm}$ de profundidade, em uma área de pousio sob pastagem natural, e posteriormente foram encaminhadas ao laboratório especializado para caracterização química (Tabela 1). O solo utilizado nesse estudo foi proveniente do município de Cruz das Almas-Ba, no campus da UFRB.

O delineamento experimental utilizado foi inteiramente casualizado, sendo composto por cinco tratamentos (Solo sem adubação, com adubação referência, -N, -P e -K), com cinco repetições, totalizando 25 unidades experimentais. A dose referência (DR) foi estabelecida pelo Manual de recomendação e adubação de Minas Gerais (Ribeiro et al., 1999), fornecendo assim N: 30 P: 100 e K: $80 \mathrm{Kg} \mathrm{ha}^{-1}$.

Utilizou-se como substrato $3 \mathrm{~kg}$ de solo em cada vaso plástico, o qual apresentava capacidade de $3 \mathrm{dm}^{3}$, posteriormente foi adicionado ao solo as doses de nitrogênio, fósforo e potássio, sendo empregada uma mistura de ureia, super triplo e cloreto de potássio, nas quantidades definidas pelos tratamentos e homogeneizados. As distribuições foram realizadas seguindo o arranjo estatístico, no qual um dos nutrientes era omitido enquanto os demais são mantidos no nível referencial.

A semeadura foi realizada diretamente nos vasos plásticos, sendo distribuída cinco sementes por vasos, uma semana após, foi feito o desbaste para garantir a homogeneidade do material, permanecendo apenas uma planta por recipiente.

Aos 30 dias após a aplicação dos tratamentos foram avaliados os parâmetros de crescimento, altura das plantas (ALT), com a utilização de uma fita métrica, medindo da base da planta até a inserção do último par de folhas e o número de folhas $(\mathrm{NF})$, em cada tratamento foi obtido através da contagem direta e diâmetro do colmo utilizando um paquímetro digital (PEIXOTO et al., 2011).

Os componentes da parte aérea e raízes das plantas de milho, foram coletados e acondicionados individualmente em sacos de papel, colocadas em estufa, com circulação forçada de ar, $60^{\circ} \mathrm{C} \pm 2^{\circ} \mathrm{C}$, até atingir peso constante. Posteriormente, após $72 \mathrm{~h}$, foram analisadas as variáveis massa seca da parte aérea (MSPA) e da raiz (MSR), utilizandose de uma balança analítica com precisão de três casas. Essas varáveis foram aferidas em $\mathrm{g}$ de fitomassa.

Tabela 1. Análise química das amostras de solo utilizadas na produção das mudas antes da aplicação dos tratamentos Cruz das Almas-Ba, 2018.

Table 1. Chemical analysis of soil samples used in seedling production before application of treatments Cruz das Almas-Ba, 2018.

\begin{tabular}{|c|c|c|c|c|c|c|c|c|c|c|c|c|}
\hline Prof. & $\mathrm{pH}$ & $\mathrm{P}$ & $\mathrm{K}$ & $\mathrm{Ca}^{2+}$ & $\mathrm{Mg}^{2+}$ & $\mathrm{Al}^{3+}$ & $\mathrm{H}+\mathrm{Al}$ & SB & $\mathrm{CTC}(\mathrm{t})$ & $\mathrm{CTC}(\mathrm{T})$ & Mo & V \\
\hline $\mathrm{Cm}$ & $\mathrm{H}_{2} \mathrm{O}$ & \multicolumn{2}{|c|}{$\mathrm{mg} / \mathrm{dm}^{3}$} & & \multicolumn{6}{|c|}{ - } & \multicolumn{2}{|c|}{$\%$} \\
\hline $0-20$ & 5,6 & 0 & 7,82 & 0,8 & 0,5 & 0 & 1,5 & 1,32 & 1,32 & 2,82 & 0,96 & 46,81 \\
\hline
\end{tabular}

Prof. = Profundidade; $\mathrm{pH}$ = Potencial hidrogeniônico; $\mathrm{P}=$ Fósforo; $\mathrm{K}=$ Potássio; $\mathrm{Ca}=$ Cálcio; $\mathrm{Mg}=$ Magnésio; $\mathrm{Al}$ = Alumínio; $\mathrm{H}+\mathrm{AL}=\mathrm{Acidez}$ potencial; $\mathrm{SB}=$ Soma de bases; $\mathrm{CTC}=$ Capacidade de troca catiônica $(\mathrm{t})$ : efetiva e $(\mathrm{T})$ : potencial; Mo = Matéria orgânica presente no solo; $\mathrm{V}=$ Saturação por bases.

\subsection{Determinação dos nutrientes da fitomassa vegetal}

As amostras foram moídas em moinho tipo Wiley, padronizado com peneira de 20 mesh e acondicionadas em sacos plásticos. Aproximadamente $0,1 \mathrm{~g}$ da massa seca da parte aérea e raízes, separadamente, foram submetidas a digestão ácida em uma mistura de 3,5 mL de ácido sulfúrico concentrado $\left(\mathrm{H}_{2} \mathrm{SO}_{4}\right)$, e $3 \mathrm{~mL}$ de peróxido de hidrogênio $\left(\mathrm{H}_{2} \mathrm{O}_{2}\right)$ a $30 \%$. O material digerido foi diluído para $100 \mathrm{~mL}$ com água destilada, obtendo-se assim, o extrato para realização das análises de nitrogênio $(\mathrm{N})$, fósforo $(\mathrm{P})$ e

Nativa, Sinop, v. 8, n. 4, p. 567-571, jul./ago. 2020. 
potássio (K). Os teores de $\mathrm{N}$ foram determinados pelo método espectrofotométrico do fenol-hipoclorito, os de $\mathrm{P}$ pelo método espectrofotométrico do molibdo-vanadato e os de $\mathrm{K}$ determinados por fotometria de chama (LIMA et al., 2017).

\subsection{Análise estatística}

Os resultados foram submetidos à análise estatística de variância, e em função do nível de significância pelo teste de F, a partir daí procedeu-se o teste de médias (Tukey 5\%), utilizando-se o programa estatístico SISVAR ${ }^{\circledR} \quad 5.3$ (FERREIRA, 2011).

\section{RESULTADOS}

Os resultados expressos através dos respectivos quadrados médios para o crescimento das plantas de milho, demonstrados na Tabela 2 , evidenciam que todas as variáveis analisadas sofreram efeito significativo em relação aos tratamentos estudados, isso implica dizer que ao menos um desses diferiu estatisticamente dos demais em cada parâmetro estudado.

Verificou-se que a altura e o número de folhas (Tabela 3) das plantas de milho apresentaram respostas similares, onde os tratamentos DR e as omissões de $\mathrm{N}$ e $\mathrm{K}$ não diferiram entre si, e foram significativamente superior aos demais. Para o diâmetro do colmo identificou-se que a DR e a omissão de $\mathrm{K}$ não diferiram entre si, e foram estatisticamente melhores em comparação aos outros tratamentos.

Para as variáveis de massa seca (Tabela 3) foi observado desempenho similar, os tratamentos que proporcionaram o maior acúmulo de fitomassa foram, a DR e $-\mathrm{K}$, apresentando desempenho significativo quando comparado aos demais tratamentos.

Também foi destacado que os valores de MSR foram superiores aos de MSPA.

Foi observado através dos respectivos quadrados médios para a diagnose nutricional nas plantas de milho (Tabela 4), que todas as variáveis analisadas sofreram efeito significativo em relação aos tratamentos estudados.

Tabela 2. Resumo da análise de variância com respectivos quadrados médios para as variáveis: Altura, diâmetro do colmo, número de folhas, massa seca da parte aérea (MSPA) e massa seca de raiz (MSR), em plantas de milho cultivadas com omissões de macronutrientes Cruz das Almas-Ba, 2018

Table 2. Summary of analysis of variance with respective mean squares for the variables: Height, stem diameter, leaf number, shoot dry mass (MSPA) and root dry mass (MSR), in omitted maize plants of macronutrients Cruz das Almas-Ba, 2018.

\begin{tabular}{cccccc}
\hline Fontes de Variação & Altura $(\mathrm{cm})$ & Diâmetro do Colmo $(\mathrm{mm})$ & Número de folhas & MSPA $(\mathrm{g})$ & MSR $(\mathrm{g})$ \\
\hline Tratamentos & $270,86^{* *}$ & $133,16^{* *}$ & $4,06^{* *}$ & $53,80^{* *}$ & $152,49^{* *}$ \\
Erro & 3,44 & 2,48 & 0,20 & 1,04 & 2,62 \\
CV $(\%)$ & 13,17 & 16,98 & 8,10 & 26,47 & 20,44 \\
\hline
\end{tabular}

**Significativo á $1 \%$ de probabilidade pelo teste $\mathrm{F}$.

Tabela 3. Altura, diâmetro da haste, número de folhas, massa seca da parte aérea (MSPA) e massa seca de raiz (MSR) de plantas de milho cultivadas com omissão de macronutrientes. Cruz das Almas-Ba, 2018.

Table 3. Height, stem diameter, leaf number, shoot dry mass (SDM) and root dry mass (RDM) of macronutrient omitted maize plants. Cruz das Almas-Ba, 2018.

\begin{tabular}{|c|c|c|c|c|c|}
\hline Tratamentos & Altura (cm) & Diâmetro do colmo (mm) & Número de folhas & MSPA (g) & $\operatorname{MSR}(g)$ \\
\hline Sem adubação & $6,5 b$ & $5,7 \mathrm{~b}$ & $4,2 \mathrm{~b}$ & $0,43 c$ & $2,44 \mathrm{c}$ \\
\hline Dose referencia & $19,5 \mathrm{a}$ & $14,9 \mathrm{a}$ & $6,0 \mathrm{a}$ & $7,5 \mathrm{a}$ & $13,5 \mathrm{a}$ \\
\hline$-\mathrm{N}$ & $19,2 \mathrm{a}$ & $6,6 \mathrm{~b}$ & $6,0 \mathrm{a}$ & $4,8 b$ & $10,13 b$ \\
\hline$-\mathrm{P}$ & $5,6 \mathrm{~b}$ & $4,5 b$ & $5,0 \mathrm{~b}$ & $0,38 c$ & $1,62 \mathrm{c}$ \\
\hline$-\mathrm{K}$ & $19,7 \mathrm{a}$ & $14,8 \mathrm{a}$ & $6,4 a$ & $6,18 \mathrm{ab}$ & $11,9 \mathrm{ab}$ \\
\hline $\mathrm{CV}(\%)$ & 13,17 & 16,98 & 8,10 & 26,47 & 20,44 \\
\hline
\end{tabular}

*Letras iguais não diferem entre si nas colunas, pelo teste de Tukey a $5 \%$ de probabilidade.

Tabela 4. Resumo da análise de variância com respectivos quadrados médios para as variáveis: Nitrogênio na parte aérea ( $N$ PA) e raiz (N Raiz), Fósforo na parte aérea (P PA) e raiz (P Raiz), Potássio na parte aérea (K PA) e raiz (K Raiz), em plantas de milho cultivadas com omissões de macronutrientes. Cruz das Almas-Ba, 2018.

Table 4. Summary of variance analysis with respective mean squares for the variables: Nitrogen on shoot (N PA) and root (N Root), Phosphorus on shoot (P PA) and root (P Root), Potassium on shoot (K PA) and root (K Root) in maize plants grown with macronutrient omissions. Cruz das Almas-Ba, 2018.

\begin{tabular}{|c|c|c|c|c|c|c|}
\hline \multirow[t]{2}{*}{ Fontes de Variação } & N PA & N Raiz & P PA & P Raiz & K PA & K Raiz \\
\hline & \multicolumn{6}{|c|}{ 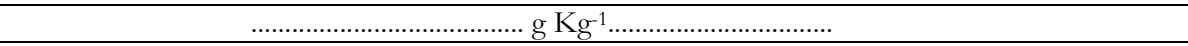 } \\
\hline Tratamentos & $76,82^{* *}$ & $33,53^{* *}$ & $0,07 * *$ & $0,34^{* *}$ & $169,15^{* *}$ & $9,62^{* *}$ \\
\hline Erro & 1,40 & 1,01 & 0,001 & 0,16 & 5,21 & 1,73 \\
\hline $\mathrm{CV}(\%)$ & 8,90 & 11,37 & 6,02 & 15,33 & 15,60 & 30,69 \\
\hline
\end{tabular}

**Significativo á $1 \%$ de probabilidade pelo teste $\mathrm{F}$.

Observou-se que a concentração de N PA (Tabela 5) foi significativamente superior quando as plantas foram cultivadas com omissão de $\mathrm{P}$, apresentando um diferencial médio de $61 \%$ em relação ao tratamento com omissão de $\mathrm{N}$.
Para os valores de N Raiz percebeu-se desempenho similar, sendo que os valores encontrados com $-\mathrm{P}$ não diferiu estatisticamente do solo sem adubação e da DR, fazendo a mesma comparação anterior esse valor cai para 58\%. 
Analisando os resultados para o teor de P nas plantas de milho, foi verificado desempenho similar nas variáveis P PA e P Raiz, onde a DR e -N não diferiram entre si, e foram significativamente superior aos demais tratamentos, destacando que os valores encontrados para $-\mathrm{K}$ não apresentaram-se estatisticamente diferentes daqueles observados na DR para P PA e -N para P Raiz. Fazendo a relação entre a MSR (Tabela 3) e o teor de P Raiz, utilizando como parâmetro a DR, percebe-se que as plantas de milho acumularam em média 11,34 $\mathrm{mg}$ de $\mathrm{P}$ em suas raízes, e 5,1 $\mathrm{mg}$ de $\mathrm{P}$ na parte aérea, totalizando $16,44 \mathrm{mg}$ de $\mathrm{P}$ na planta nesse estádio inicial de crescimento.

Para a concentração de $K$ nas plantas de milho, foi destacado que, o tratamento sem adubação apresentou-se significativamente superior aos demais. Os resultados de $\mathrm{K}$ nas raízes, mostraram que os tratamentos $-\mathrm{N}$, $-\mathrm{P}$ e $-\mathrm{K}$ proporcionaram significativos acúmulos desse nutriente em relação aos demais tratamentos.

Tabela 5. Nitrogênio na parte aérea (N PA) e raiz (N Raiz), Fósforo na parte aérea (P PA) e raiz (P Raiz), Potássio na parte aérea (K PA) e raiz (K Raiz), em plantas de milho cultivadas com omissões de macronutrientes. Cruz das Almas-Ba, 2018.

Table 5. Nitrogen in shoot (N PA) and root (N Root), Phosphorus in shoot (P PA) and root (P Root), Potassium in shoot (K PA) and root (K Root) in plants cultivated with macronutrient omissions. Cruz das Almas-Ba, 2018.

\begin{tabular}{|c|c|c|c|c|c|c|}
\hline \multirow[t]{2}{*}{ Tratamentos } & N PA & N RAIZ & P PA & P RAIZ & $\mathrm{KPA}$ & K RAIZ \\
\hline & \multicolumn{6}{|c|}{ 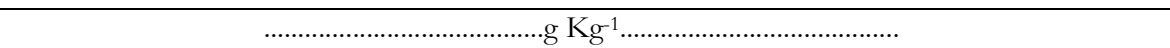 } \\
\hline Sem adubação & $14,42 b$ & $10,42 \mathrm{a}$ & $0,47 \mathrm{c}$ & $0,23 \mathrm{~d}$ & $24,81 \mathrm{a}$ & $3,57 \mathrm{bc}$ \\
\hline Dose referência & $12,73 \mathrm{~b}$ & $10,15 \mathrm{ab}$ & $0,68 \mathrm{ab}$ & $0,84 a$ & $10,71 b$ & $2,74 \mathrm{c}$ \\
\hline$-\mathrm{N}$ & $7,03 \mathrm{c}$ & $4,49 \mathrm{c}$ & $0,73 \mathrm{a}$ & $0,80 \mathrm{ab}$ & $13,93 b$ & $6,11 \mathrm{a}$ \\
\hline$-\mathrm{P}$ & $17,61 \mathrm{a}$ & $10,74 \mathrm{a}$ & $0,47 \mathrm{c}$ & $0,40 \mathrm{c}$ & $12,36 \mathrm{~b}$ & $5,33 \mathrm{ab}$ \\
\hline$-K$ & $14,69 \mathrm{~b}$ & $8,49 \mathrm{~b}$ & $0,64 b$ & $0,65 b$ & $11,32 \mathrm{~b}$ & $3,67 \mathrm{abc}$ \\
\hline $\mathrm{CV}(\%)$ & 8,9 & 11,37 & 6,02 & 15,33 & 15,60 & 30,69 \\
\hline
\end{tabular}

*Letras iguais não diferem entre si nas colunas, pelo teste de Tukey a $5 \%$ de probabilidade.

\section{DISCUSSÃO}

A altura e o número de folhas caracterizam-se como variáveis muito importantes, pois estão diretamente relacionadas ao porte da planta, permitindo que o vegetal consiga captar a energia luminosa e consequentemente produzir fotoassimilados. Em relação ao diâmetro do colmo, Peixoto et al., (2011) afirmaram que esse parâmetro está intimamente relacionado ao acúmulo de reservas, além de garantir a sustentação da planta.

Os resultados encontrados neste trabalho evidenciam a importância do fornecimento dos macronutrientes primários no crescimento inicial de plantas de milho, sendo assim, Taiz et al. (2017) afirmaram que o acúmulo da fitomassa seca representa o saldo entre os fotoassimilados e a respiração.

Quanto aos maiores valores de MSR, é destacado que, apresenta relação direta à fase inicial do crescimento vegetal, porque existe a necessidade de expansão do sistema radicular para potencializar a absorção de água e sais minerais.

Resultados semelhantes foram encontrados por Gondim et al. (2016), ao estudarem o crescimento inicial de plantas de milho com omissões de macronutrientes em solução nutritiva, destacando que a omissão de $\mathrm{N}$ e $\mathrm{P}$ principalmente afetaram significativamente as variáveis altura, $\mathrm{n}^{\mathrm{o}}$ de folhas, diâmetro da haste, massa seca e área foliar.

Considerando uma população de 60 mil plantas por hectare em 30 dias de cultivo, observa-se de acordo os valores apresentados (Tabela 3) que provavelmente o incremento da MSPA para as plantas sem o fornecimento dos adubos minerais seria de $25,8 \mathrm{Kg} \mathrm{ha}^{-1}$, em contrapartida, com o fornecimento desses, esse valor aumenta significativamente para $450 \mathrm{~kg} \mathrm{ha}^{-1}$, evidenciando assim a importância desses nutrientes na fase inicial do crescimento vegetal e no acumulo de fitomassa, possivelmente associada a potencialização da atividade fotossintética.

No tocante a significância da diagnose nutricional em todos os parâmetros estudados, fica evidenciado assim que os macronutrientes primários podem comprometer $\mathrm{O}$ balanço nutricional do vegetal.

$\mathrm{O} \mathrm{N}$ e o $\mathrm{P}$ podem interagir de forma sinérgica, ambos auxiliam na produção vegetal, quando o suprimento do P é limitado, a assimilação de $\mathrm{N}$ normalmente é comprometida, afetando assim a translocação de $\mathrm{N}$ para a parte aérea, devido a restrição do transporte via simplasto da raiz para o xilema, acarretando na acumulação de $\mathrm{N}$ no sistema radicular (Fernandes, 2006).

Em relação a significância da concentração de $\mathrm{K}$ na testemunha, foi evidenciado que existiu uma baixa requisição desse nutriente na fase inicial de crescimento vegetal, provavelmente a quantidade presente no solo e as reservas cotiledonares foram suficientes para suprir a necessidade do vegetal. Provavelmente ocorram reações antagônicas na absorção dos sais minerais fazendo com que nesse período a suplementação de $\mathrm{K}$ não se mostre eficiente.

Segundo Fernandes (2006), diversas pesquisas com K nos solos brasileiros tem demonstrado respostas não acentuadas a fertilização desse nutriente, isso deve estar atrelado a fatores como, contribuições de formas não trocáveis, presença de minerais fontes de $\mathrm{K}$, entre outros.

De maneira geral foi perceptível que no crescimento inicial das plantas de milho, o elemento que se apresentou como limitante foi o $\mathrm{P}$, sendo este elemento constituinte da parede celular e principalmente nas atividades metabólicas como moeda energética. A deficiência de $\mathrm{P}$ pode promover ao vegetal o acúmulo de antocianina, que conferem as folhas uma coloração avermelhada e verde-escura, além de comprometer severamente todo metabolismo, podendo ser fator limitante na floração, embonecamento e enchimento de grãos. Plantas de milho crescidas sob condições de baixa disponibilidade de $\mathrm{P}$ podem apresentar severa inibição a assimilação fotossintética do $\mathrm{CO}_{2} \mathrm{em}$ folhas, assim como redução nas atividades das enzimas ribulose 1,5-bifosfato carboxilase, piruvato ortofosfato diquinase e fosfoenolpiruvato carboxilase, essas que são indispensáveis no metabolismo de plantas C4 e na via redutiva das pentoses fosfato (TAIZ et al., 2017).

\section{CONCLUSÕES}

O crescimento inicial das plantas de milho é prejudicado com a omissão de nitrogênio e fósforo. 
O potássio é o elemento que se apresenta em maior concentração no estádio inicial de crescimento, seguido do nitrogênio e fósforo.

\section{AGRADECIMENTOS}

O presente trabalho foi realizado com apoio da Coordenação de Aperfeiçoamento de Pessoal de Nível Superior - Brasil (CAPES) - Código de Financiamento 001.

A Universidade Federal do Recôncavo da Bahia pelas condições oferecidas.

\section{REFERÊNCIAS}

CARMO, D. L.; TAKAHASHI, H. Y. U.; SILVA, C. A.; GUIMARÃES, P. T. G. Crescimento de mudas de cafeeiro recém-plantadas: efeito de fontes e doses de fósforo. Coffee Science, Lavras, v. 9, n. 2, p. 196-206, 2014.

CONAB_COMPANHIA NACIONAL DE ABASTECIMENTO. Safra Brasileira de Grãos 2018/19 - Segundo Levantamento da CONAB. Disponível em: http:/ / www.conab.gov.br/conteudos.php?a $=1253 \& \mathrm{t}=2$ Acesso em: 19 de novembro 2018.

ELEMER, W. H.; DATNOFF, L. E. Mineral Nutrition and Suppression of Plant Disease. Encyclopedia of Agriculture and Food Systems, v. 4, 231-244, 2014. DOI: http://dx.doi.org/10.1016/b978-0-444-52512.00251-5

FERNANDES, M. S. Nutrição mineral de plantas. Viçosa, MG. Sociedade Brasileira de Ciência do solo. 2006, p.432.

FERREIRA, D. F. Sisvar: a computer statistical analysis system. Ciência e agrotecnologia, v. 35 n. 6, p. 10391042, 2011.

GONDIM, A. R. D. O.; MELLO, P. R.; ALVES, A. U.; FONSECA, I. M. Eficiência nutricional do milho cv. BRS 1030 submetido à omissão de macronutrientes em solução nutritiva. Ceres, Viçosa, v. 57, n. 4, 2010. DOI: http://dx.doi.org/10.1590/S0034-737X2010000400017

GONDIM, A. R. D. O.; PRADO, R. M.; FONSECA, I. M.; ALVES, A. U. Crescimento inicial do milho cultivar brs 1030 sob omissão de nutrientes em solução nutritiva. Ceres, Viçosa, v. 63, n. 5 2016. DOI: http://dx.doi.org/10.1590/0034-737x201663050016

IBGE_Instituto Brasileiro de Geografia e Estatística. Produção agrícola municipal - 2017. Disponível em: https://sidra.ibge.gov.br/tabela/1612\#resultado. Acesso em: 30 de novembro de 2018.

LIMA, J. C.; OLIVEIRA, U. C.; SANTOS, A. R.; SOUZA, A. A.; SOUZA, G. S. Diagnose nutricional de Lippia alba (Mill) N. E. Br. cultivada sob proporções de amônio e nitrato e ambientes de luz. Revista de Ciências Agrárias, Lisboa, v. 40, n.4, p. 770-776, 2017.

MCGRATH, J. M.; SPARGO, J.; PENN, C. J. Soil Fertility and Plant Nutrition. Encyclopedia of Agriculture and Food Systems, v. 5 p. 166-184. DOI: http://dx.doi.org/10.1016/b978-0-444-52512-3.002497

PEIXOTO, C. P.; CRUZ, T. V.; PEIXOTO, M. F. S.; Análise quantitativa do crescimento de plantas: Conceitos e Prática. Enciclopédia Biosfera, Goiânia, v. 7, p. 5176, 2011.

RIBEIRO, A. C.; GUIMARÃES, P. T. G.; ALVAREZ, V. H. Recomendações para o uso de corretivos e fertilizantes em Minas Gerais, $5^{a}$ aproximação. 1999, p. 359.

TAIZ, L.; ZEIGER, E.; MOLLER, I.M.; MURPHY, A.; Fisiologia e Desenvolvimento Vegetal. 6. ed. 2017, p.858.

VIEIRA, C. R.; DOS SANTOS W.; OSCARLINA, L.; SCARAMUZZA, J. F. Omissão de macronutrientes no desenvolvimento de mudas de mogno africano. Revista Ecologia e Nutrição Florestal, Santa Maria, v. 2, n. 3, p. 72-83, $2014 . \quad$ DOI: http://dx.doi.org/10.5902/2316980X1604 